\title{
A case of Guillain-Barré syndrome (GBS) presenting with acute urinary retention and T6 sensory level
}

\author{
Authors: Ching Soong Khoo, ${ }^{A}$ Alyaa Hassan Ali, ${ }^{B}$ Rabani Remli ${ }^{C}$ and Hui Jan $\operatorname{Tan}^{\mathrm{D}}$
}

\begin{abstract}
Guillain-Barré syndrome (GBS) is an acute immune-mediated demyelinating disease. Early recognition of this disease is crucial as it can progress to life-threatening conditions such as respiratory failure or autonomic dysfunction. Typical clinical manifestations of GBS include progressive weakness of the limbs, bulbar, facial muscles and ophthalmoplegia. Sensory level and bladder dysfunction are more suggestive of acute myelopathy. We report a case of GBS presenting with acute urinary retention and T6 sensory level, which was successfully treated with plasma exchange.
\end{abstract}

KEYWORDS: Guillain-Barré syndrome, plasmapheresis, sensory level, bladder dysfunction, nerve conduction study

\section{Case presentation}

A 41-year-old Myanmarese man with no past medical history presented with a 3-day history of progressive lower limb weakness and urinary retention. There was no back pain, upper limb weakness, diplopia, dysphagia or shortness of breath. He denied any recent history of trauma to the spine. Apart from chronic alcohol consumption, he denied drug abuse or sexual promiscuity. Three days prior to this current emergency visit, he had presented with fever and symptoms of upper respiratory tract infection. He had then been discharged with outpatient treatment.

Upon review, he was alert, conscious and not in respiratory distress. His presenting vitals were as follows: blood pressure $130 / 78 \mathrm{mmHg}$, heart rate 92 beats per minute (bpm), temperature $37^{\circ} \mathrm{C}$, peripheral capillary oxygen saturation $\left(\mathrm{SpO}_{2}\right) 96 \%$ on room air. His lower limb examination revealed a muscle power of $0 / 5$, absent tendon reflexes at the patellas and ankles, bilateral downgoing plantar response and reduced sensation to soft touch and pin prick from T6 and below. The upper limb examination was unremarkable with intact cranial nerves and negative cerebellar signs. There was no paraspinal tenderness. Abdominal

Authors: ${ }^{A}$ neurology fellow and physician, Universiti Kebangsaan Malaysia Medical Centre, Kuala Lumpur, Malaysia; ${ }^{B}$ physician, Universiti Kebangsaan Malaysia Medical Centre, Kuala Lumpur, Malaysia; ${ }^{\mathrm{C}}$ consultant neurologist, Universiti Kebangsaan Malaysia Medical Centre, Kuala Lumpur, Malaysia; ${ }^{\mathrm{D}}$ consultant neurologist, Universiti Kebangsaan Malaysia Medical Centre, Kuala Lumpur, Malaysia examination revealed a dull mass at the suprapubic region. Other physical examinations including the cardiovascular, respiratory systems and skin were unremarkable. His full blood count, renal profile with electrolytes, liver function, cardiac enzymes, blood gas and random blood sugar tests were within normal limits. His electrocardiogram (ECG) was normal. His forced vital capacity (FVC) was approximately $25 \mathrm{~mL} / \mathrm{kg}$.

\section{Diagnosis}

Acute transverse myelitis was the most pertinent diagnosis in view of his rapid onset of paraplegia with a sensory level and bladder dysfunction. GBS ought to be considered for his areflexia in the lower limbs. Spinal cord compression would be an important differential, but absence of back pain made this less likely. A diagnosis of nutritional neuropathy like thiamine deficiency was also considered in this case for his chronic alcoholism.

\section{Initial management and prognosis}

A urinary catheter was inserted promptly and almost a litre of urine was drained. He was given an immediate dose of intravenous (IV) thiamine. A neurology registrar counselled the patient for urgent magnetic resonance imaging (MRI) of his spine. He refused the MRI adamantly due to his financial constraints and lacking insight into the severity of his illness. A nerve conduction study (NCS) was ordered and he was placed in the ward pending other work-up.

\section{Case progression and outcome}

The patient tested negative for HIV, syphilis, malaria, dengue fever, leptospirosis, Herpes virus, Epstein-Barr virus, Cytomegalovirus and mycoplasma infections. His B12, folate, thiamine levels and thyroid function were all normal. While waiting for the NCS (day 2 of admission), the patient complained of breathlessness and became dyspnoeic. Lungs revealed equal vesicular breath sounds. Immediate arterial blood gas showed type I respiratory failure. A portable chest X-ray film was grossly normal. He became better with supplemental oxygen and finally consented to an urgent spine MRI. Neurologically, he remained the same. However, the patient was not monitored closely for FVC in the ward.

About 1 hour after coming back from the MRI suite, he became increasingly short of breath and did not respond to non-invasive 
ventilation. Repeated blood gas revealed type II respiratory failure. Endotracheal intubation was performed immediately. He was subsequently nursed at the intensive care unit (ICU).

The MRI of the whole spine was normal. NCS detected absent $F$-waves in both common peroneal nerves and tibial nerves with absent $\mathrm{H}$-reflexes bilaterally. In view of the negative spine imaging, positive neurophysiological study, paraplegia with areflexia and respiratory failure, a diagnosis of GBS was formulated. After a lengthy discussion with the patient's friends, we decided to treat him with plasmapheresis given its affordability.

After receiving the first session of plasmapheresis, we started noticing clinical improvement, namely present bilateral ankle reflexes and slight active movement of his ankles. He tolerated the treatment well and was able to be extubated after 5 sessions of plasma exchange. He suffered no dysautonomia throughout. Cerebrospinal fluid (CSF) taken on day 7 of illness revealed albuminocytologic dissociation. Other CSF analyses were normal. The patient was discharged with a nasogastric tube (due to his bulbar dysfunction) and bladder catheter after 2 weeks of hospitalisation. Upon clinic visit at 6 weeks, he showed complete recovery with no neurological sequelae (GBS Disability Scale at 0). Repeated NCS was normal (Table 1).

\section{Discussion}

GBS is an acute monophasic paralysing immune-mediated polyneuropathy, which is the most frequent case of acute flaccid paralysis world-wide. Overall incidence of GBS is approximately 1-2 cases per 100,000 per year. ${ }^{1}$ GBS is usually associated with preceding infections with organisms like Campylobacter

\begin{tabular}{|c|c|c|c|}
\hline & $\begin{array}{l}\text { Day } 2 \text { in } \\
\text { the ward }\end{array}$ & $\begin{array}{l}\text { Day } 9 \text { in } \\
\text { the ward }\end{array}$ & $\begin{array}{l}6 \text { weeks at } \\
\text { the clinic }\end{array}$ \\
\hline $\begin{array}{l}\text { F-wave } \\
\text { (Right tibial nerve) } \\
\text { (Normal value }<56 \text { ms) }\end{array}$ & Absent & $51.9 \mathrm{~ms}$ & $46.65 \mathrm{~ms}$ \\
\hline $\begin{array}{l}\text { F-wave } \\
\text { (Left tibial nerve) } \\
\text { (Normal value }<56 \text { ms) }\end{array}$ & Absent & $49.6 \mathrm{~ms}$ & $46.9 \mathrm{~ms}$ \\
\hline $\begin{array}{l}\text { F-wave } \\
\text { (Right common } \\
\text { peroneal nerve) } \\
\text { (Normal value <56 ms) }\end{array}$ & Absent & $\begin{array}{l}67.3 \mathrm{~ms} \\
\text { (Prolonged) }\end{array}$ & $48.55 \mathrm{~ms}$ \\
\hline $\begin{array}{l}\text { F-wave } \\
\text { (Left common peroneal } \\
\text { nerve) } \\
\text { (Normal value < } 56 \text { ms) }\end{array}$ & Absent & $\begin{array}{l}58.4 \mathrm{~ms} \\
\text { (Prolonged) }\end{array}$ & $53.5 \mathrm{~ms}$ \\
\hline $\begin{array}{l}\text { H-reflex } \\
\text { (Right tibial nerve) } \\
\text { (Normal value <32 ms) }\end{array}$ & Absent & Absent & $22.15 \mathrm{~ms}$ \\
\hline $\begin{array}{l}\text { H-reflex } \\
\text { (Left tibial nerve) } \\
\text { (Normal value }<32 \text { ms) }\end{array}$ & Absent & Absent & $28.5 \mathrm{~ms}$ \\
\hline
\end{tabular}

jejuni, Cytomegalovirus, Epstein-Barr virus, HIV and Zika virus. There are other triggering factors for developing GBS such as trauma, surgery, bone marrow transplant and systemic illnesses like systemic lupus erythematosus (SLE). ${ }^{2}$ The cardinal clinical features of GBS include progressive symmetrical muscle weakness accompanied by absent or reduced deep tendon reflexes. A diagnosis of GBS is made by the clinical manifestations, CSF analysis and electrophysiological studies. Bladder dysfunction at onset with a sensory level is more suggestive of acute myelopathy and makes a diagnosis of GBS doubtful.

There are many variants of GBS. Acute inflammatory demyelinating polyneuropathy (AIDP) is the most common variant, which represents up to $90 \%$ of all cases of GBS. AIDP carries a better prognosis compared with the axonal types as peripheral nerve remyelination occurs relatively rapidly over weeks. Only a small majority of patients with AIDP will develop severe secondary axonal degeneration. Our patient suffered from AIDP variant of GBS, which might explain his good recovery and normal NCS upon follow-up.

GBS involves the multifocal demyelination at the level of the nerve roots. ${ }^{3,4}$ It is hypothesised that our patient had initial inflammation at the nerve roots up to the level of T6 giving rise to the symmetric T6 sensory loss clinically. The absent F-waves and $\mathrm{H}$-reflexes explain the involvement of the proximal segment (the nerve roots in our case). A similar case was described by Alfahad and Kelly in 2014 as the first case of GBS presenting with a sensory level as an initial manifestation, which was successfully treated with immunoglobulin. ${ }^{5}$

Recognition of GBS and subsequent initiation of immunotherapy and supportive care is critically important due to its associated risks of developing respiratory failure and dysautonomia., ${ }^{6,7}$ Respiratory failure is common, as $15-30 \%$ of GBS patients will require ventilation. Frequent FVC measurements, looking for bulbar dysfunctions or inability to clear secretions are crucial in monitoring GBS patients for respiratory failure. Immunotherapy such as plasma exchange or intravenous immunoglobulin (IVIG) hastens recovery in GBS patients. The effects of both treatments are equally beneficial, while combining both does not result in a better outcome. In our case, plasma exchange was opted for because of its affordability for the patient.

\section{Key learning points:}

> Sensory level and bladder dysfunction, although unusual, may present as the initial manifestations in GBS. In doubtful cases, an urgent spine MRI and eletrophysiological studies will be helpful

> Rapid onset of paraplegia with a clearly defined sensory level and bladder or bowel dysfunction is suggestive of acute myelopathy; however, areflexia should raise the possibility of GBS

> Vigilance for respiratory failure like frequent FVC monitoring is crucial in patients presenting with progressive muscle weakness

> IVIG and plasma exchange are both equally effective in treating GBS with atypical presentations as demonstrated in both our case reports.

\section{Consent}

Written consent has been obtained from the patient for publication of the clinical details in this article. 


\section{References}

1 Yuki N, Hartung HP. Guillain-Barré Syndrome. N Engl J Med 2012;366:2294-304.

2 Ropper AH. The Guillain-Barré Syndrome. N Engl J Med 1992:326:1130.

3 Kimura J. Proximal versus distal slowing of motor nerve conduction velocity in the Guillain-Barré syndrome. Ann Neurol 1978;3:344.

4 Gordon PH, Wilbourn AJ. Early electrodiagnostic findings in GuillainBarré syndrome. Arch Neurol 2001;58:913.

5 Alfahad TB, Kelly J]. Pure T7 sensory level as an isolated manifestation of Guillain-Barré syndrome. J Clin Neuromuscul Dis 2014;15:102-4.
6 Zochodne DW. Autonomic involvement in Guillain-Barré syndrome: a review. Muscle Nerve 1994;17:1145.

7 Hughes RA, Wijdicks EF, Benson E et al. Supportive care for patients with Guillain-Barré Syndrome. Arch Neurol 2005;62:1194.

Address for correspondence: Dr Ching Soong Khoo, Department of Medicine, Universiti Kebangsaan Malaysia Medical Centre, Jalan Yaacob Latif, Bandar Tun Razak, Cheras Kuala Lumpur 56000, Malaysia.

Email: chingsoongkhoo@gmail.com 